\title{
INVESTIGAÇÃO, SIMULAÇÃO E DIAGNÓSTICOS SOBRE O CONTROLE DE ACIONAMENTO DE UMA BOBINADEIRA DE AÇO INOXIDÁVEL*
}

\author{
Igor Barros Mairinck ${ }^{1}$ \\ Lenin Martins Ferreira Morais ${ }^{2}$ \\ Seleme Isaac Seleme Júnior ${ }^{2}$
}

\section{Resumo}

Este trabalho consiste num estudo investigativo, no campo teórico, visando levantar informações de processo, modelagem e simulações do sistema de controle da linha de Preparação de Bobinas 1 (PB1), processo em batelada de corte e bobinamento de aços inoxidáveis da APERAM. A partir deste modelo simulado, foi realizada avaliação de desempenho e sugeridas alterações do controle e novos parâmetros do controlador de bobinamento, de forma a contribuir para a melhora do desempenho das malhas de controle e do processo real. Foram analisadas as interações entre os componentes do circuito de controle e os impactos no desempenho global do sistema no domínio do tempo e da frequência. Este conhecimento adquirido propiciou sugestões de melhoria do comportamento do sistema.

Palavras-chave: Corte de aço inoxidável; Controle analógico; Simulação; Modelagem.

\section{RESEARCH, SIMULATION AND DIAGNOSTICS ON THE DRIVE CONTROL OF A STAINLESS STEEL WINDER}

\begin{abstract}
This work consists of an investigative study, in theory, aimed at raising process information, modeling and simulations of the control system of the Coils Preparation line 1 (PB1), a batch process in stainless steels cutting and winding services. From this simulation model, a performance evaluation was conducted and changes of the controller and new parameters of the winding controller were suggested, in order to contribute to the improvement of the performance of control and the real process loops. The interactions between the control circuit components and the impact on the overall performance of the system in the time and frequency domains were analyzed. This acquired knowledge was reversed in improving the system behavior suggestions.

Keywords: Stainless steel cutting; Analog control; Simulation; Modeling; Transfer functions.
\end{abstract}

Graduado em Engenharia Elétrica, Mestre em Engenharia Elétrica, Supervisor de Manutenção, Siderurgia, Acabamento de Aços Inoxidáveis, APERAM, Timóteo, MG, Brasil.

2 Departamento de Engenharia Eletrônica, Grupo de Eletrônica de Potência, UFMG, Belo Horizonte, MG, Brasil. 


\section{INTRODUÇÃO}

Este trabalho foi desenvolvido em uma linha de produção da APERAM.

A PB1 tem por função o corte de pontas conforme metragem indicada nos mapas de inspeção das bobinas à quente (BQ), soldar tira-líder, aparar bordas tanto para processo como para adequar à largura final exigida pelos clientes, dividir bobinas, cortar peso máximo em bobinas destinadas à expedição, retirar amostras, fazer marcação contínua, recuperar bobinas acidentadas nos laminadores de bobinas ou recuperar defeitos de bordas oriundos da laminação de Tiras à Quente (LTQ) ou do primeiro recozimento. A linha de produção PB1 é composta dos subconjuntos básicos principais identificados na figura 1.

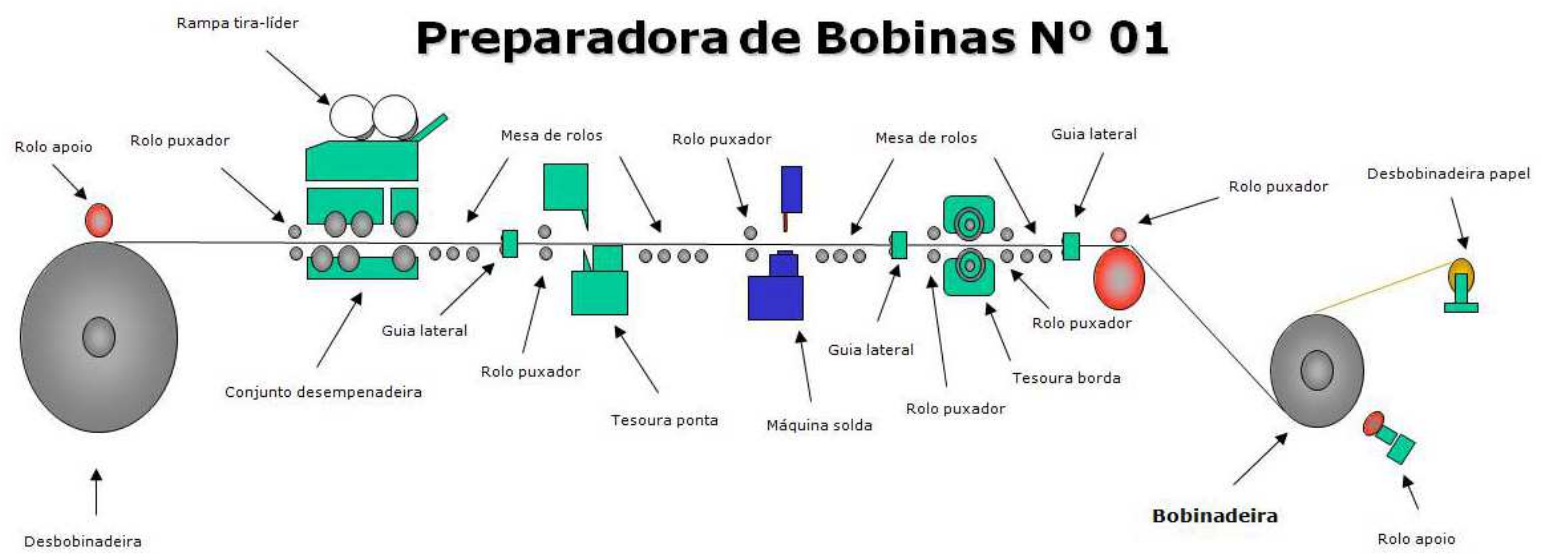

Figura 1. Topologia básica da PB1 - Principais componentes.

Todos os sub-conjuntos são acionados por motores c.c. com excitação independente e conversores analógicos. O conjunto final da linha PB1, a bobinadeira, está registrado em foto na figura 2 vista pelo lado da operação (LO).

Trata-se, no acionamento, de um motor c.c. do fornecedor "GE" com $245 \mathrm{cv}$ (ou 180 kW) de potência, tensão nominal ("Va") de $500 \mathrm{~V}$ e corrente nominal ("la") de $400 \mathrm{~A}$ na armadura. A PB1 é uma linha com componentes eletrônicos antigos e obsoletos e que vem apresentando desempenho operacional instável, paradas não programadas, limitação operacional do equipamento.

Os objetivos gerais são: Estudar as malhas de controle do motor c.c. de $180 \mathrm{~kW}$ do conjunto "bobinadeira" do equipamento "PB1" da APERAM através de modelagem e simulação computacional. Modelar o seu comportamento, simulá-lo em ferramentas computacionais disponíveis e propor controles que permitam melhorar o desempenho de produção.

As publicações nacionais relacionadas a seguir, serviram de referência para este trabalho. Em [1], foi abordado o controle de velocidade de motor de corrente contínua (motor c.c.), alimentado por conversor reversível c.a/c.c. Em [2] estão os ajustes de comissionamento do equipamento na época da instalação. Em [3], foi realizado estudo do controle de Conversores Estáticos Reversíveis c.a/c.c. com excitação separada, fixa e com limitação da corrente e de sua derivada. No trabalho de [4], procedeu-se com a Modelagem e Simulação do Processo de Bobinamento na Laminação de tiras para Aplicações de Controle. O estudo de [5] apresenta a modelagem, análise e controle de um sistema de bobinamento de tiras de aço produzidas através de lingotamento contínuo do tipo twin roll. 


\section{MATERIAIS E MÉTODOS}

\subsection{O Processo do Equipamento Preparadora de Bobinas no1 (PB1)}

Na tabela 1 estão registradas as especificações básicas da linha da PB1 conforme projeto original.

Tabela 1: Especificações do fabricante da preparadora de bobina no1 (PB1).

\begin{tabular}{|c|c|}
\hline \multicolumn{2}{|c|}{ Preparadora } \\
\hline \hline Fabricante & DMS \\
\hline Tipo & $\begin{array}{c}\text { Stainless Steel, cold or Hot rolled } \\
\text { AISI 200-300-400 }\end{array}$ \\
\hline Início de operação & 1977 \\
\hline Capacidade (Ton/ano) & 60.000 \\
\hline Acionamento da preparadora & Tensão auxiliar: $120 \mathrm{~V}-60 \mathrm{~Hz}$ \\
\cline { 2 - 2 } & Tensão do motor AC: $440 \mathrm{~V} \mathrm{-60} \mathrm{Hz}$ \\
\hline Velocidade (m/min) & 125 \\
\hline
\end{tabular}

\begin{tabular}{|c|c|c|}
\hline \multicolumn{3}{|c|}{ Bobinas } \\
\hline \hline & Nominal & Real \\
\hline Espessura (mm) & $\begin{array}{c}6,00 \text { (Máximo) até } \\
0,50 \text { (mínimo) }\end{array}$ & $\begin{array}{c}7,93 \text { (Máximo) até } \\
0,50 \text { (mínimo) }\end{array}$ \\
\hline Largura máxima (mm) & 1270 & 1270 \\
\hline Diâmetro ext. máx. (mm) & 1830 & 1830 \\
\hline Diâmetro interno (mm) & 610 & 610 \\
\hline Peso (ton) & 15 & 25 \\
\hline
\end{tabular}

O conversor de armadura usado na PB1 para acionamento da bobinadeira é o modelo Jistor da Série 400 do fabricante Jeumont-Schneider. O controle de velocidade do motor c.c. se dá com excitação separada através de uma ponte monofásica controlada e alimentado na armadura por um conversor c.a./c.c. com duas pontes de Graëtz em anti-paralelo, com limitação da corrente e de sua derivada.

A filosofia de acionamento é "fluxo proporcional ao diâmetro". A figura 2 apresenta o diagrama de blocos geral da malha de controle. Existe um circuito de controle da corrente e tensão de campo e outro para regulação da corrente e tensão de armadura. Nota-se a presença de duas malhas de controle em cascata (ou em série) sendo que a malha interna é a de corrente e a externa de velocidade (tensão).

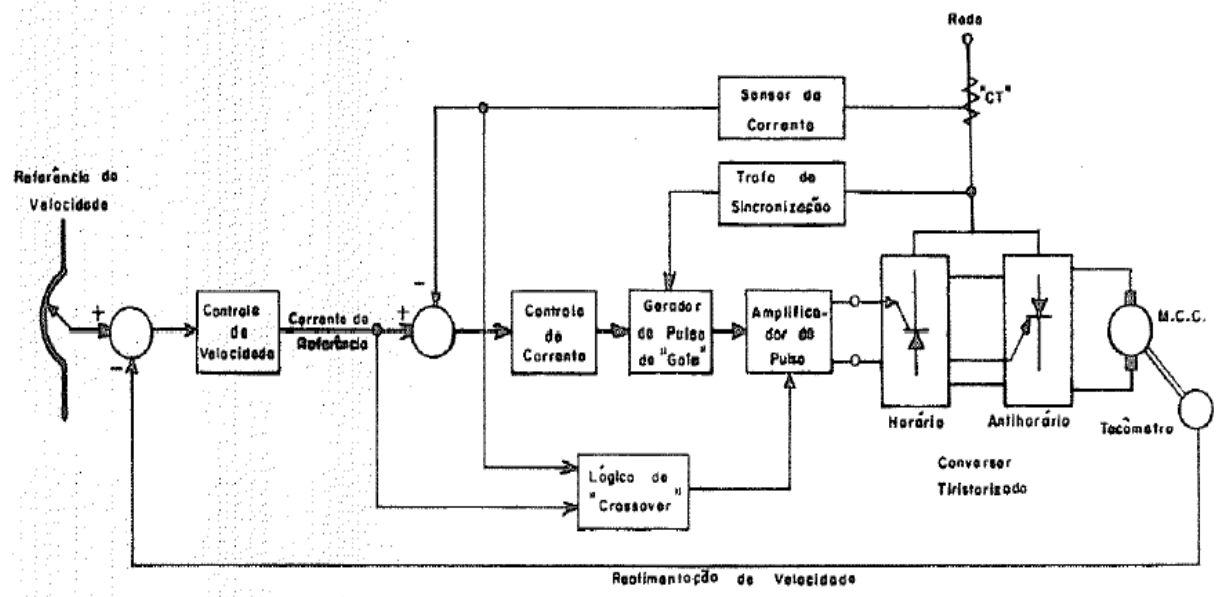

Figura 2. Topologia das malhas de controle

\section{RESULTADOS E DISCUSSÃO}

\subsection{Representação da Malha de Controle Global}

Estão representados nas figuras 3 e 4 os respectivos circuitos modelados da malha de controle global com as entradas 1 e 2 . 

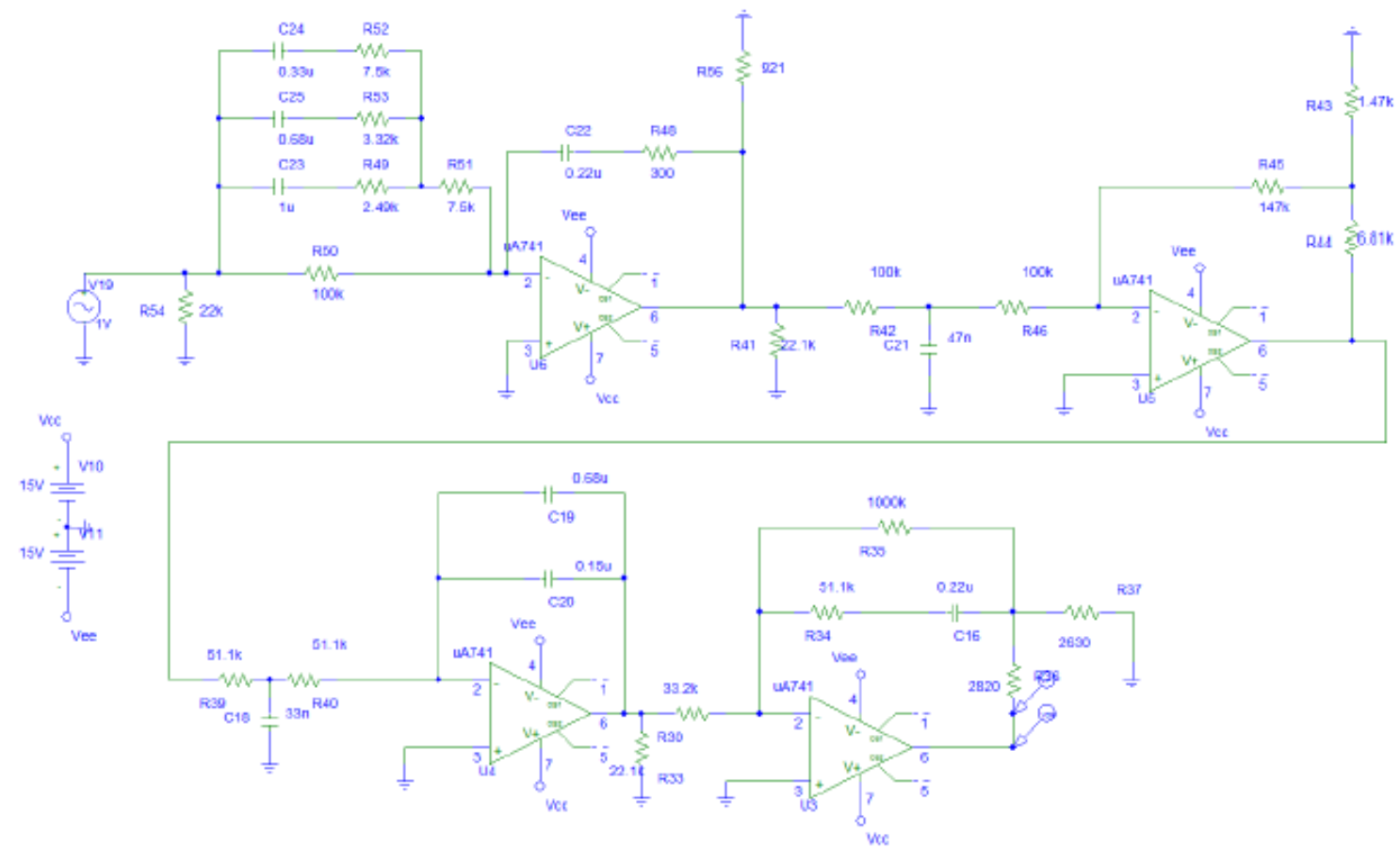

Figura 3. Diagrama esquemático para a Malha Global - Entrada 1
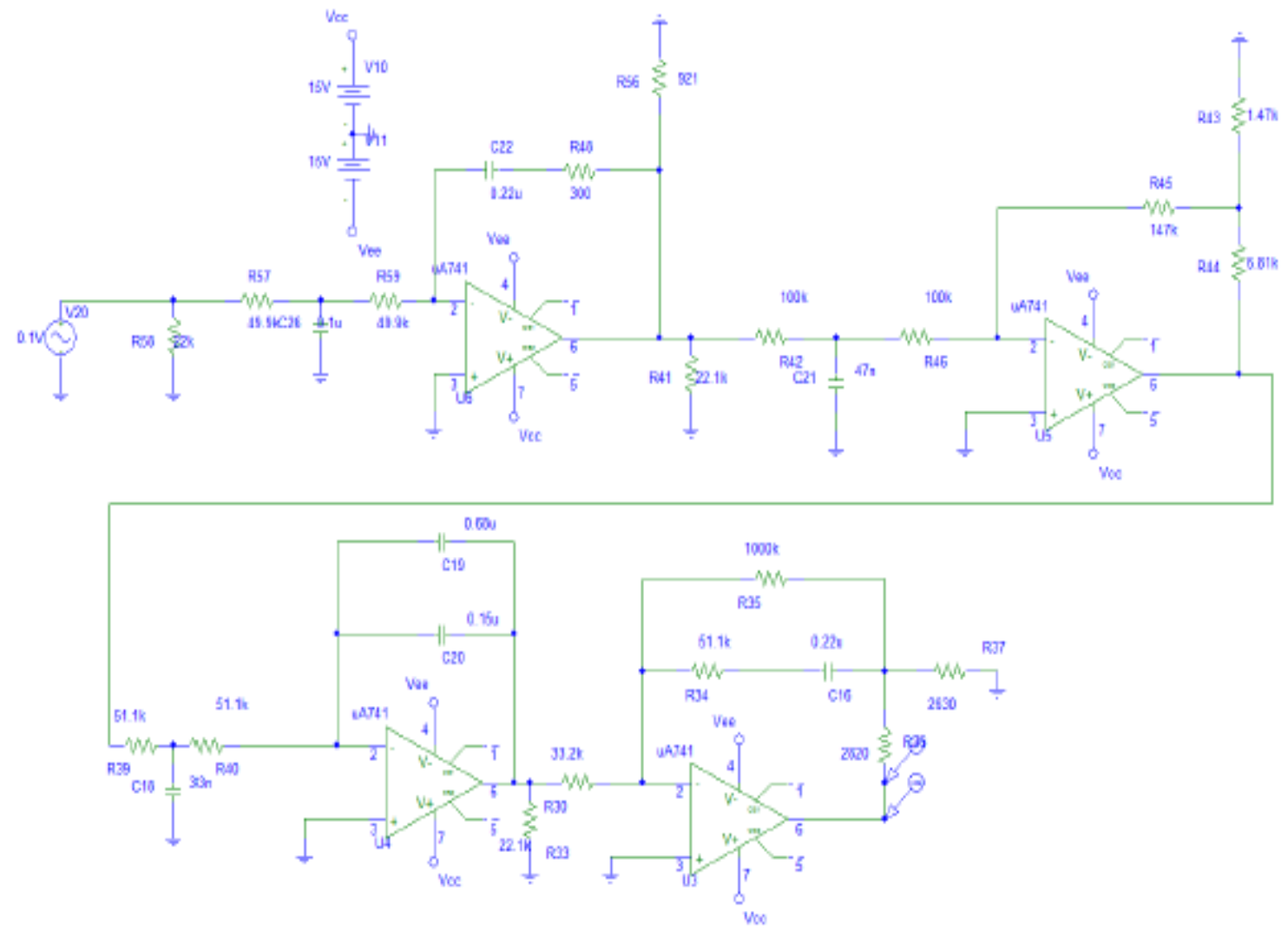

Figura 4. Diagrama esquemático para a Malha Global - Entrada 2

A figura 5 apresenta a resposta em frequência para os dois circuitos que modelam a malha global para as entradas 1 e 2 . 

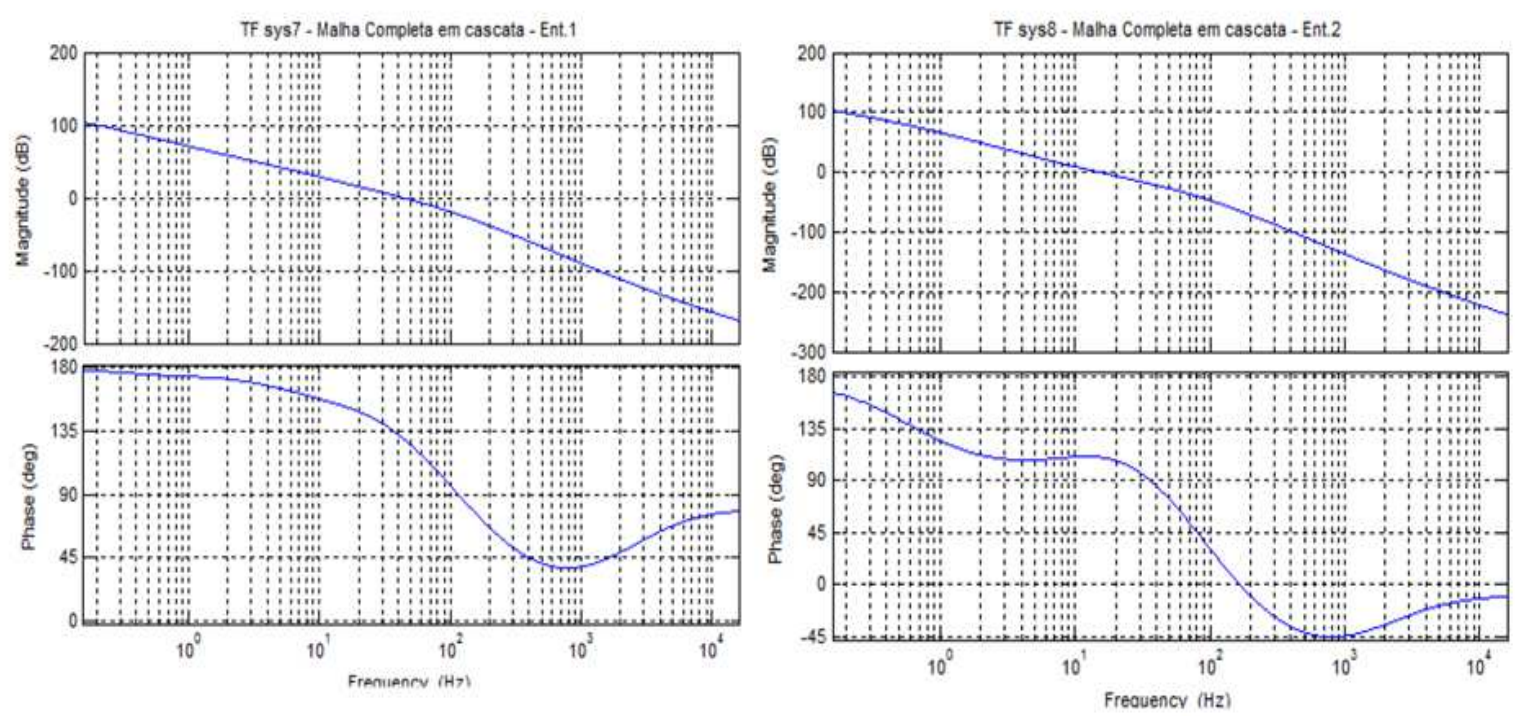

Figura 5. Simulação - Malha Global - Entradas 1 e 2

As equações que representam as Funções de Transferências dos circuitos supra citados estão registradas nas equações 1 e 2.

$$
\begin{array}{ll}
F T_{s 6}=\frac{-41.1 s^{5}-5.8 e 5 s^{4}+3.7 e 12 s^{3}+5.2 e i 6 s^{2}+4.4 e i 8 s+1.8 e 19}{s^{7}+1.1 e 6 s^{6}+1.9 e 9 s^{5}+6.72 e i 1 s^{4}+3.36 e 13 s^{3}+1.22 e i 4 s^{2}} & \text { Equação } 1 \\
F T_{s 7}=\frac{-1437 s^{4}-1.6 e 7 s^{3}+1.29 e 14 s^{2}+1.498 e 18 s+1.19 e 20}{s^{7}+1.1 e 6 s^{6}+2.2 e 9 s^{5}+1.2 e 12 s^{2}+2.02 e 14 s^{3}+7.79 e 14 s^{2}} & \text { Equação 2 }
\end{array}
$$

\subsection{Avaliação de Desempenho}

Foi simulado e analisado o controlador analógico face a variações paramétricas consideradas importantes. São analisadas as interações entre os componentes do circuito de controle e os impactos no desempenho global do sistema no domínio do tempo e da frequência. De posse das FT's, passou-se para a simulação de todo o sistema no programa PSIM, incluindo modelos empíricos aproximados da prática em função dos dados disponíveis em placa e manual do equipamento, para o conversor, a carga e sensores.

\subsubsection{Simulação de Perturbações na medição de velocidade}

O procedimento para testar a simulação de perturbação no tacogerador é iniciado com a aplicação de soma de sinal de $0,02 \mathrm{~V}$ amplitude de pico (ou $2 \%$ de ripple) com frequência de $1 \mathrm{~Hz}$ ao sinal original do tacogerador. Para as próximas etapas o sinal de referência foi mantido inalterado, porém a frequência da perturbação somada ao sinal do taco foi aumentada em etapas. O resultado da simulação está disposto na figura 6. 


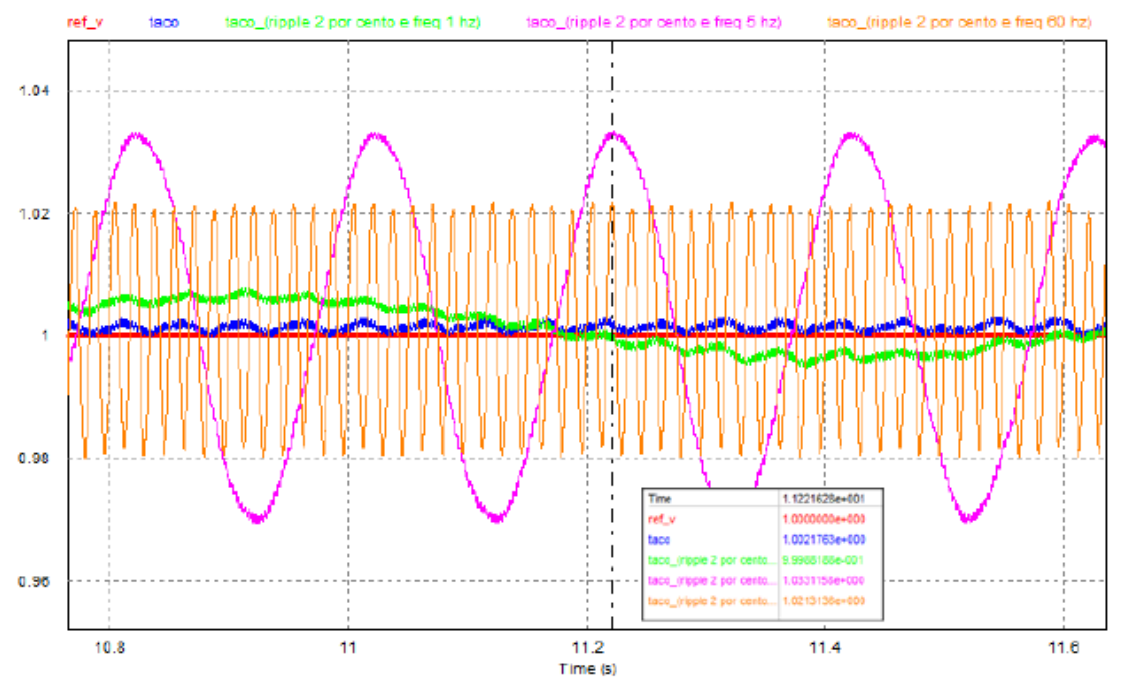

Figura 6. Interferências de freqüências distintas no sinal de realimentação de velocidade

Pela figura 7 é possível perceber que para determinada faixa de frequência a malha de controle funciona como um filtro passa-baixas. A perturbação de $5 \mathrm{~Hz}$ é amplificada em $67,5 \%$ e a perturbação de $60 \mathrm{~Hz}$ não sofre alteração. Como tentativa de aumentar a rejeição à perturbação do sistema a este tipo de ruído característico na malha de medição de velocidade, foi simulado um aumento na capacitância da malha de velocidade. $O$ valor passou de 0,22 uF para o máximo permitido pela placa que é de $1 \mathrm{uF}$, conferindo aumento de 4,54 vezes. A perturbação injetada foi de 0,1 $\mathrm{V}$ de amplitude e a frequência de $5 \mathrm{~Hz}$ foi mantida. $\mathrm{O}$ resultado apresentado na figura 8 realça o benefício do aumento da capacitância.
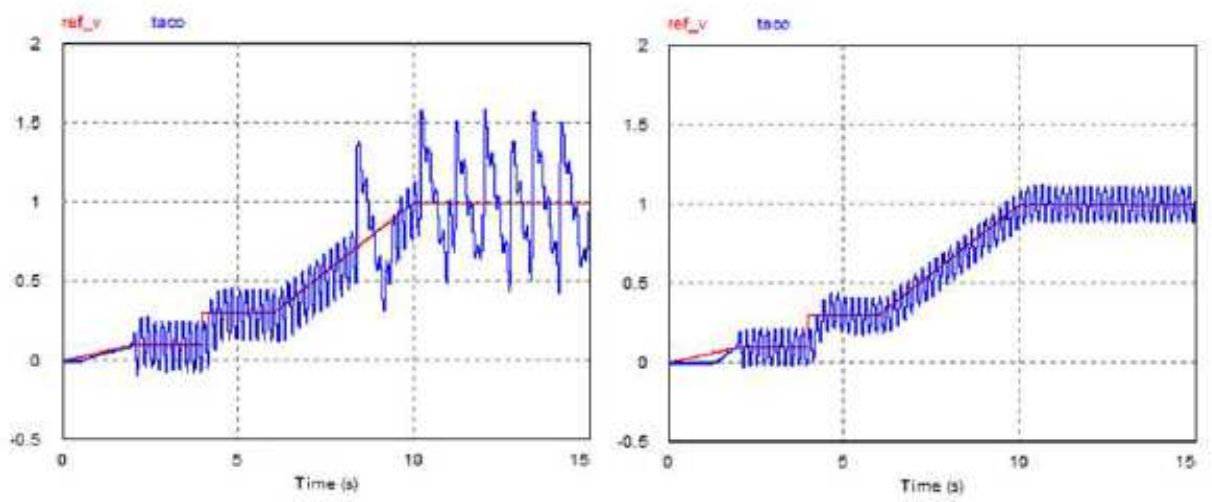

Figura 7. Resultado do aumento do parâmetro integrador do controlador

Como no caso da PB1 a velocidade de resposta não é uma característica crítica, muitas das vezes é preferível aumentar a rejeição do sistema, pois o efeito de "batimento" é muito mais danoso à máquina elétrica e ao sistema de transmissão. Portanto, esta ação de bloqueio de alterar os "straps" da placa, para aumentar o Ki (ganho integral) é válida para atenuar o efeito de perturbação na medição de velocidade e pôde ser comprovada na simulação realizada.

\subsubsection{Simulação do efeito do laço anti-windup da malha de corrente}

Nesta seção o objetivo foi comprovar o benefício da utilização do laço anti-windup da malha de corrente. Neste sentido, foram simuladas as duas situações possíveis, a primeira com o laço compensatório anti-windup e a segunda sem este laço. 
A referência foi apropriadamente escolhida de forma que gerasse variação rápida o suficiente para levar o controle ao seu limite de saturação. Desta forma, ao sinal de rampa foi somado um sinal em forma triangular, de $0,5 \mathrm{~V}$ pp e com frequência de $2 \mathrm{~Hz}$. A rampa é a modelagem da situação de um bobinamento, variando de 0 à 1 num intervalo de 15 segundos e permanecendo neste patamar durante 5 segundos. Conforme registrado na figura 8 .

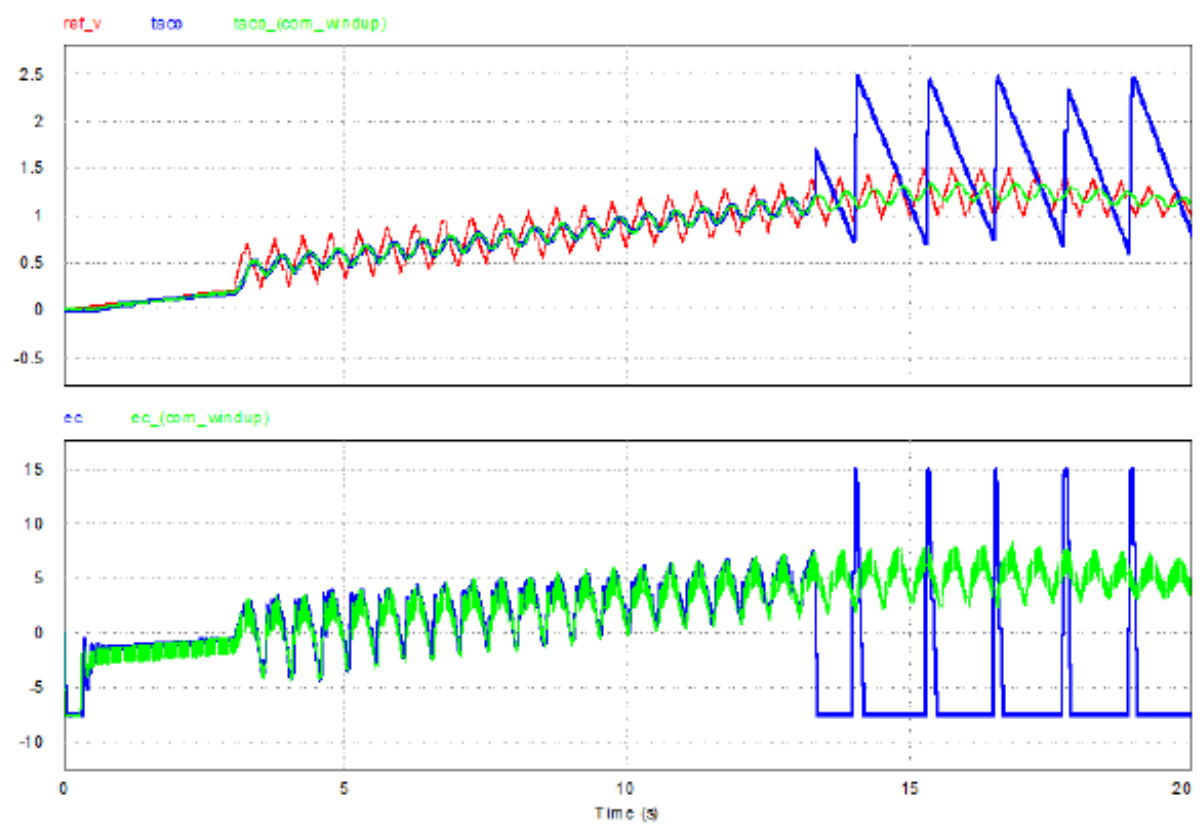

Figura 8. Resultado com (verde) e sem a malha "Anti windup" (azul)

O comportamento dos circuitos com e sem o laço anti-windup é bem semelhante até a referência alcançar o valor próximo a 1. A partir do qual, o circuito sem o laço antiwindup degrada o desempenho de forma notável quanto aos valores da variável controlada (gráfico superior) devido ao fato do sinal de saída da malha de controle saturar (gráfico inferior).

\subsubsection{Simulação da variação paramétrica}

Nesta seção é buscado o conhecimento a cerca de variações de parâmetros analógicos discretos dos PIDs clássicos. Esta variação pode ser causada por diversos fatores, seja por um desgaste de componentes com o tempo, ou pela variação com temperatura, ou simplesmente devido ao fato de que o parâmetro não foi precisamente determinado ou foi alterado indevidamente ao longo de sua vida útil.

A estratégia de simulação neste caso emulou a perda gradual de capacitância da malha de velocidade, englobando a faixa de valores de 1 _ $F$ à $1500 \mathrm{pF}$, que é justamente a faixa selecionável pelos straps na placa analógica "MDNX1". A resposta do sistema para três valores de capacitância [1 uF; 0,22 uF; 0,08 uF] está representada na figura 9. 


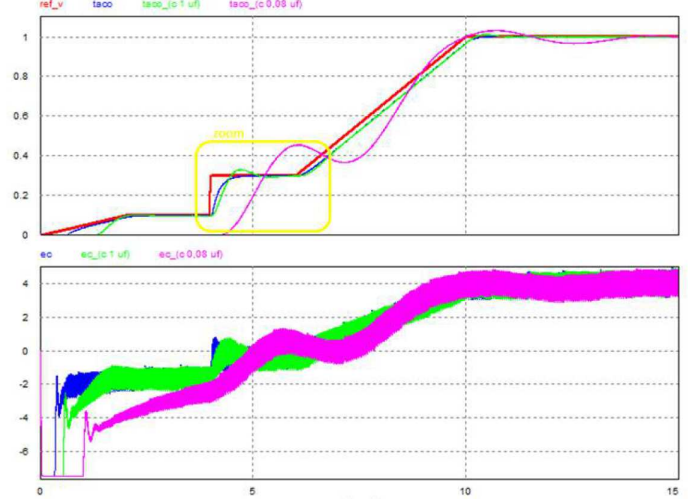

Figura 9.1. Resultado - Variações do capacitor

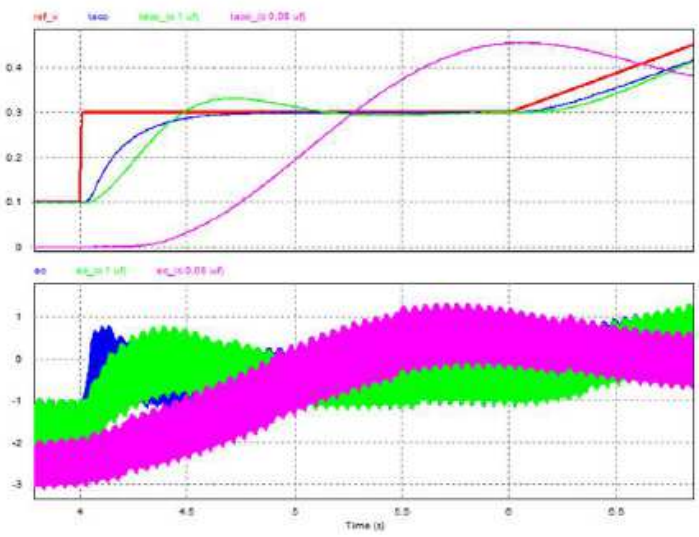

Figura 9.2 Detalhe na seleção

A próxima etapa da simulação objetivou evidenciar variações maiores de capacitância, atingindo valores extremos da faixa de ajuste, sendo os valores simulados para capacitâncias de 4,7 uF, 0,22 uF e 1500 pF. O resultado está exposto na figura 10. No gráfico superior está a referência de velocidade e as diferentes curvas de medição de velocidade. No gráfico inferior estão as diferentes saídas do controlador, sinal "ec" para cada capacitor utilizado nas três simulações.

É possível perceber que a perda de capacitância de um capacitor das malhas de controle é suficiente para alterar a dinâmica no tempo da resposta do sistema, aumentando o erro em regime e tornando o sistema mais lento, chegando até mesmo a tirá-la da região de estabilidade. Este aspecto evidenciado na simulação reforça o fato observado na prática de reajustes e re-sintonias de malhas analógicas antigas, sendo que, por muitas vezes é necessário substituir a placa analógica velha por placa nova ou recuperada em laboratório.

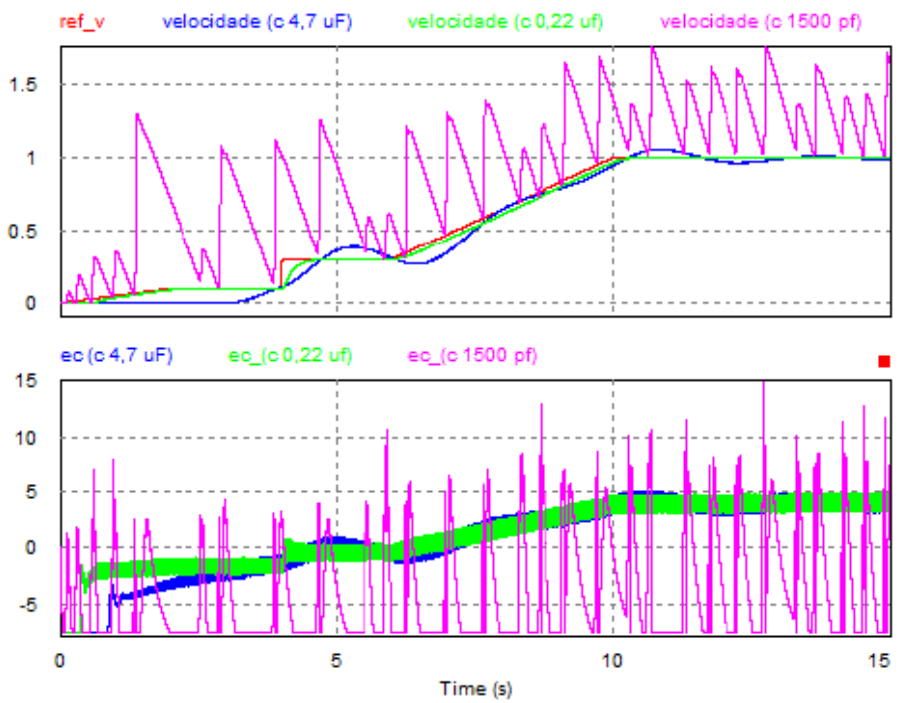

Figura 10. Curvas em resposta à variações extremas da capacitância $(C=4,7 F(A z u l), 0,22 F($ Verde) e $1500 \mathrm{pF}$ (Magenta)

\subsubsection{Simulação de avanço-atraso de fase}

Foram simuladas alterações na parte de avanço e atraso de fase da entrada da malha de velocidade e avaliar seus impactos. Foi adotada a estratégia de simular removendo cada ramo do avanço de fase da entrada do sinal do tacogerador, um de cada vez, até ser totalmente eliminado ficando apenas a entrada do taco com o seu 
ganho padrão. As curvas resultantes foram plotadas no mesmo gráfico, representado na figura 11.
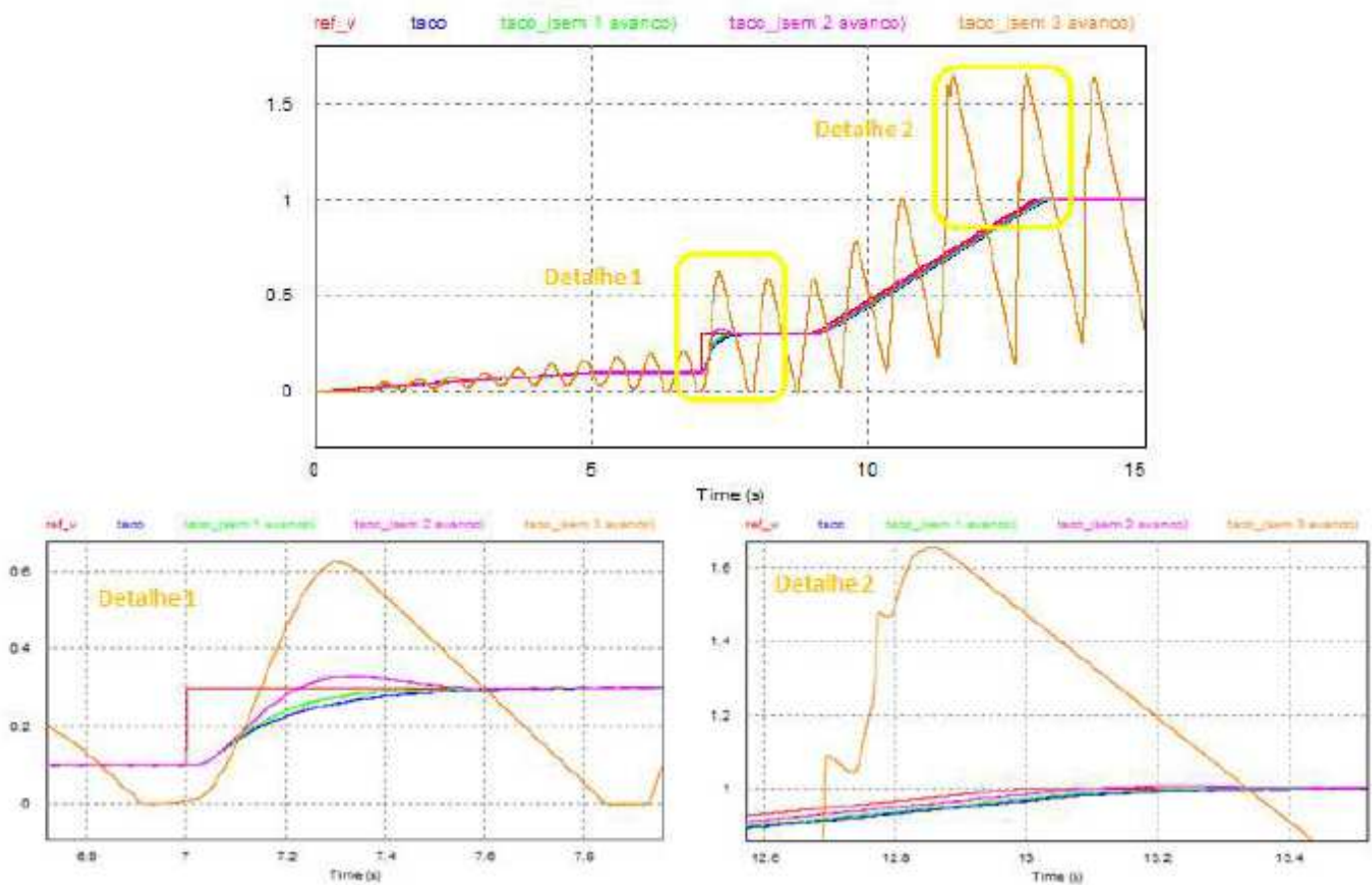

Figura 11. C Alterações nos avanços de fase. (Em Vermelho: Referência, Em Azul: topologia original, Em Verde: sem 1 compensador em avanço, Em Magenta: Sem 2 compensadores e Em Laranja: Sem compensação alguma)

Pela figura 11 nota-se que a remoção dos straps e consequentemente das compensações de avanço de fase, deixa o sistema mais rápido, contudo, leva ao aumento de oscilações face à excitação em forma de degrau. Na figura 12 é registrada o o resultado da simulação do efeito individual de cada laço de avanço de fase da entrada da velocidade medida na malha de controle de velocidade. Em cada simulação, apenas um laço estava presente.

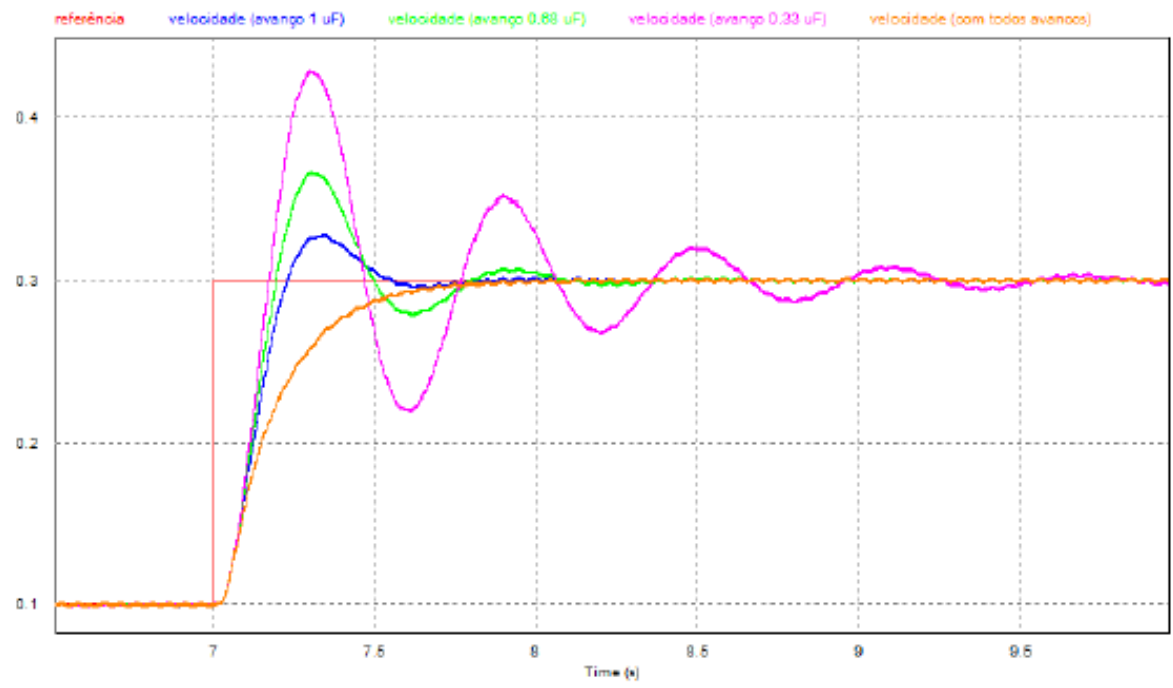

Figura 12. Alterações individuais nos avanços de fase. (Em Vermelho: Referência, Em Azul: Avanço ( $C=1 \mathrm{uF})$, Em Verde: Avanço ( $C=0,68 \mathrm{uF})$,Em Magenta: Avanço $(C=0,33 \mathrm{uF})$ e Em Laranja: Avanço (com todos os laços). 
A maior contribuição é dada pelo laço de avanço com o capacitor de maior valor, isto é, 1 uF. Portanto, a curva azul que possui o melhor desempenho é conseguida pela contribuição individual do referido laço, ao passo, que a menor contribuição é dada pelo laço com capacitância de 0,33 uF na curva de cor Magenta. O resultado ideal é conseguido apenas com a contribuição concomitante dos três laços, sendo representada pela curva em laranja.

\subsubsection{Simulação da variação dos potenciômetros das malhas de controle}

Os resultados foram impressos na figura 13, para seis pontos de operação na faixa de 0,05 à 0,9 , sendo que 0,05 o potenciômetro está com o valor mínimo e 0,9 no valor máximo.

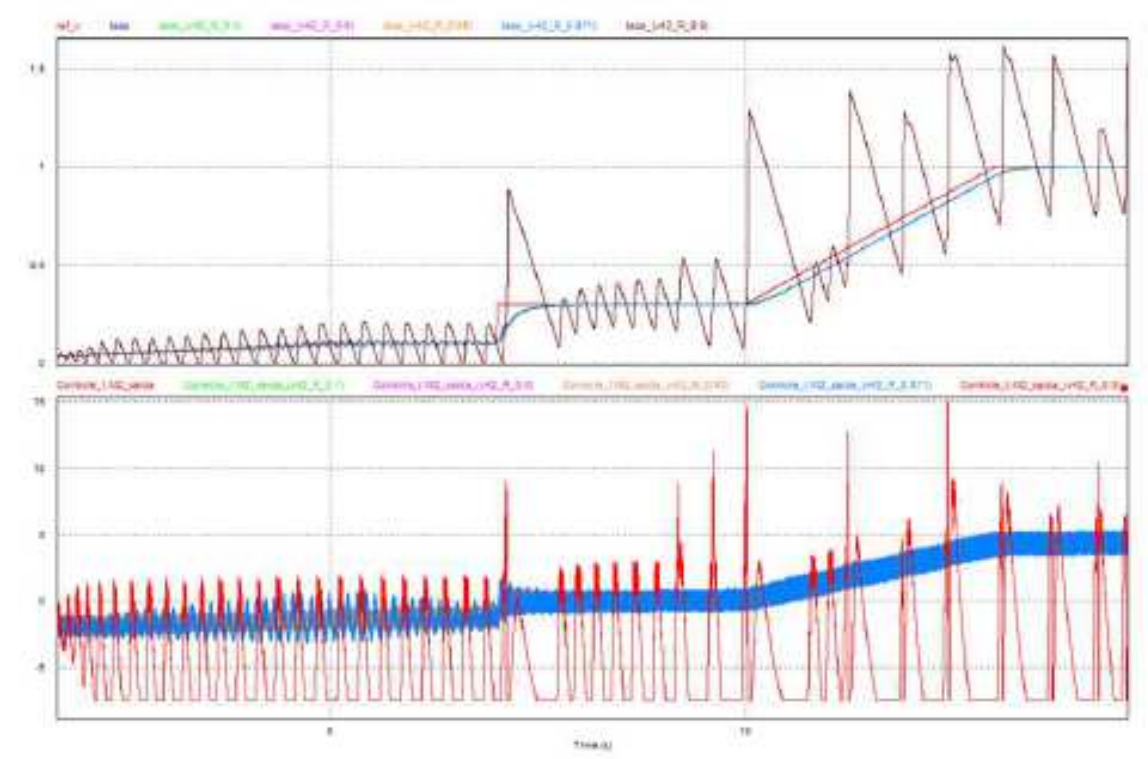

Figura 13. Simulação variando o potenciômetro da malha de velocidade e seu impacto.

\section{CONCLUSÃO}

Todo o trabalho de investigação, levantamento de informação, detalhamento de circuitos e simulação do modelo proposto serviram de base para obter o conhecimento do sistema de bobinamento e principalmente, o sistema de controle e se reverteu em sugestões de contribuições para melhoria do comportamento do sistema e para o melhor ajuste dos controladores.

Além disso, a partir do conhecimento dos padrões de respostas do sistema, estes podem ser compilados e aplicados em algoritmos de análise computacional para prever diagnósticos de falhas em estágio incipiente. Foi evidenciada a necessidade de conferência dos ajustes do controle e quando necessário, realizar a manutenção das placas.

\section{Agradecimentos}

À APERAM pela oportunidade de trabalho e estudo. 


\section{REFERÊNCIAS}

1 Vieira, A. E. (1977). Estudo dos conversores estáticos reversíveis c.a/c.c. Master's thesis, Escola de Engenharia UFMG

2 Jeumont-Schneider (1976). Manual técnico - funcionamento e manutenção do equipamento elétrico.

3 Carlos, J. (1980). Controle de velocidade de motor de corrente contínua, alimentado por conversor reversível c.a/c.c. Master's thesis, Escola de Engenharia, PPGEEUFMG.

4 Filho, J. D., Figueiredo, B. R., and Helman, H., editors (1996). Modelamento e Simulação do Processo de Bobinamento na Laminação de tiras para Aplicações de Controle.

5 Lima, F. (2001). Modelagem, analise e controle de um sistema de bobinamento de tiras de aço. Master's thesis, Engenharia de São Carlos da Universidade de São Paulo. 